\title{
Hospital Queuing-Recommendation for Time Prediction Algorithm in Bigdata
}

\author{
Hanumantha $\mathrm{K} \mathrm{N}^{1}$, Sunitha $\mathrm{S}^{2}$ \\ M.Tech (CSE), Department of CSE, RBYMEC, Cantonment, Ballari ${ }^{1}$ \\ M.Tech (Lecturer), Department of CSE, RBYMEC, Cantonment, Ballari²
}

\begin{abstract}
Viable patient line administration to limit persistent holdup postponements and patient congestion are one of the significant difficulties confronted by doctor's facilities. Pointless and irritating sits tight for long stretches result in a significant human asset, time loss and increment dissatisfaction persisted by victims. Propose a PTTP calculation to foresee the sitting tight time to individual therapy errand for a victim. We utilize the practical patient information from different doctor's facilities to get a patient treatment time display for each errand. In light of this vast scale, practical dataset and the therapy time to every victim in the present line of every errand are anticipated. In light of the anticipated holding up time and Hospital Queuing-Recommendation (HQR) framework are created.
\end{abstract}

Index Terms: Apache Spark, huge information, distributed computing, healing center lining suggestion, quiet treatment time forecast.

\section{INTRODUCTION}

Most doctors' facilities are stuffed and need viable patient line administration. Persistent line administration and hold up time expectation shape a testing and confused occupation on the grounds that every patient may require distinctive stages/operations, different tests were taken for example X-beams or a CT examine, diabetic level or blood test, small surgeries, amid therapy. Above mentioned stages/operations as therapy undertakings or assignments in this paper. Every therapy assignment will have fluctuating time prerequisites to the individual victim, which sets aside a few minutes' expectation and suggestion exceedingly convoluted. A victim is undergoing these tests as per his condition Effective patient line administration to limit quiet hold up postponements, tolerant congestion is one of the significant difficulties confronted by healing centers. Pointless and irritating sits tight for long stretches result in the considerable human asset, time loss and increment disappointment persisted by victims.

In light of the expansive scale and a practical dataset of the therapy time to the individual victim in the present line of every assignment are anticipated. In light of the anticipated holding up time and Hospital Queuing-Recommendation (HQR) framework are produced. As of now, the most healing facilities are stuffed, need viable patient line administration. Tolerant line administration and sit tight for time forecast shape a testing and entangled occupation in light of the fact that every patient may require diverse stages operations, different tests were taken for example Xbeams or a CT examine, diabetic level or blood test, small surgeries, amid therapy.

Persistent Therapy Time Prediction sample is prepared in view of healing centers' chronicled information. The holding up time of every patient treatment assignment can be anticipated by the PTTP demonstrate and the whole of all patients holding up times in the present line. As indicated by every patient's asked for treatment errands, a Hospital QueuingRecommendation (HQR) framework prescribes a productive and advantageous treatment arrange with the slightest sitting tight time for the patient. PTTP calculation and an HQR framework. Considering the ongoing necessities, gigantic information, and many-sided quality of the framework, we utilize huge information and distributed computing samples for proficiency, versatility. PTTP calculation are prepared in view of an enhanced RF calculation to individual therapy assignment, holding up each time undertaking were anticipated in light of the prepared PTTP demonstrate

\section{RELATED WORK}

To enhance the exactness of the data examination with nonstop alternatives, various advancement methods for grouping and relapse calculations region unit anticipated

Self-versatile enlistment of relapse trees: Another calculation for incremental development of double relapse trees is exhibited. This calculation, called SAIRT, adjusts the instigated demonstrate when confronting information streams including obscure flow, similar to progressive and sudden capacity float, changes in specific locales of the capacity, commotion, and virtual float. It likewise handles both typical and numeric properties. The proposed calculation can 
consequently adjust its inside parameters and model structure to acquire new examples, contingent upon the present elements of the information stream.

SAIRT can screen the handiness of hubs and can overlook cases from chose districts, putting away the staying ones in nearby windows related to the leaves of the tree. On these conditions, current relapse strategies require a watchful setup relying upon the elements of the issue. Experimentation recommends that the proposed calculation gets preferred outcomes over current calculations when managing information streams that include changes with various paces, clamor levels, inspecting conveyance of illustrations, and halfway or finish changes of the fundamental capacity

Parallel supported relapse trees for Web look positioning: Angle Boosted Regression Trees (GBRT) has the back and forth movement best in class learning perspective for machine learned web-look situating - a space scandalous for extensive informational collections. Propose a strategy for parallelizing readiness of GBRT. Method parallelizes development of the each relapse trees, works utilizing ace laborer worldview takes after. Information is parceled among the laborers. At every emphasis, the laborer abridges its information parcel utilizing histograms. The ace processor utilizes these to fabricate one layer of a relapse tree, and after that sends this layer to the laborers, enabling the specialists to manufacture histograms for the following layer. Our calculation precisely coordinates cover amongst correspondence and calculation to accomplish great execution.

Since this approach depends on info assignation and needs slightly live of correspondence, it sums up to spread and shared memory machines, and additionally mists. we have a tendency to show trial comes concerning on each shared memory machines and bunches for 2 vast scales the online ask for to position informational indexes. We have a tendency to exhibit that the misfortune inexactitude motivated thanks to the bar chart estimation within the relapse tree creation is created up for through marginally a lot of profound trees. Later, we have a tendency to see no vast misfortune inexactitude on the Yahoo in foal indexes and slightly change inexactitude for the Microsoft LETOR information. Moreover, on shared memory machines, we have a tendency to acquire much idealize direct accelerate with up to around forty-eight centers on the substantial informational indexes. On taken memory machines, we have a tendency to get a speed of twenty-five with thirty-two processors. thanks to info assignation our approach will scale to considerably larger informational collections, on that one will reasonably expect significantly higher speedups.

Correlation based part criterion in the multi-branch choice tree: A standout amongst the most generally utilized prescient models in the arrangement is the choice tree (DT). The undertaking of a DT is to guide perceptions to target values. In the DT, each branch speaks to a run the show. A control resulting is the leaf of the branch and its predecessor is the conjunction of the components. Most connected calculations in this field utilize the idea of Information Entropy and Gini Index as the part standard when constructing a tree. In this paper, another part basis to construct DTs is proposed. Apart paradigm indicates the tree's best part factor and also the variable's edge for the further part. Utilizing the thought from established Forward Selection technique and its improved forms, the variable having the biggest total relationship with the objective esteem is picked as the best part factor at every hub. At that point, augmenting the edge between classes in a bolster vector machine (SVM) is utilized to locate the best arrangement edge on the chose variable.

This method will execute recursively at every hub, until achieving the leaf hubs. An official choice tree has a shorter stature than past techniques, which successfully decreases pointless factors and the time required for grouping of future information. Unclassified districts are likewise produced under the proposed technique, which can be deciphered as preference or burden. The recreation comes about exhibit a change in the produced choice tree contrasted with past strategies.

A parallel framework execution of information mining order and relapse tree (CART) calculation on a multiFPGA framework: Information mining is another field of code engineering with an in-depth sort of utilizations. it'll probably concentrate data from vast knowledge sets in an exceedingly human-reasonable structure and example are selection trees. During this article we tend to introduce inventive, elite, framework level engineering to the Classification and Regression Tree (CART) calculation, a standout amongst the foremost crucial and generally utilised calculations within the data mining zone. Our projected engineering abuses correspondence to the selection variable level, was utterly actualized, assessed on a innovative superior reconfigurable stage and the Convey HC- 1 server that elements four FPGAs, a multicore processor.

The FPGA-based execution are incorporated with the generally utilized "part" programming library of the R extend thus on provide the most utterly sensible reconfigurable framework that may touch upon real big-ticket databases. The projected framework, named HC-CART framework, accomplishes Associate in Nursing execution speeding of up to 2 requests of greatness contrasted with understood single-strung data mining programming stages, as an example, woodhen and therefore the R stage. It to boot beats comparable instrumentality frameworks that actualize components 
of the whole application by an invitation of size. At long last, we tend to demonstrate that the HC-CART framework offers higher execution speeding than another projected parallel programming usage of alternative tree development calculations.

Another structure for appropriated boosting calculation: Another structure is building boost classifier on appropriated databases. The principle thought of our strategy is to use the parallelism of conveyed databases. At each round of the calculation, each site forms its own information locally, and figures all required data. A middle site will gather data from all locales and construct the worldwide classifier, which is then a classifier in the group. This worldwide classifier is likewise utilized by each conveyed site to process required data for the following round.

By rehashing this procedure, we will have an outfit of classifier from appropriated database that is practically indistinguishable to the one based on general information. The examination comes about demonstrate that the precision of our proposed strategy is practically equivalent to the exactness when applying the boosting calculation to the entire dataset.

Fundamental commitments amid this paper are abridged as takes after.

PTTP calculations are proposed in view of enhanced RF calculation. Entirety of all patients' likely therapy times in the present line. HQR frameworks are proposed in view of anticipated holding up time. Therapy suggestions with an effective, helpful therapy arrange the slightest sitting tight time is prescribed to an individual victim.

Persistent Therapy Time Prediction sample calculation, HQR framework is parallelized on the Apache Spark cloud stage at NSCC to accomplish previously mentioned objectives. Broad healing facility information is put away in the Apache HBase, a parallel arrangement are utilized with the Map Reduce, Resilient Distributed Datasets (RDD) programming sample

\section{PROPOSED SYSTEM}

We concentrate on helping designs finish their treatment errands in an anticipated time and helping doctor's facilities plan every treatment undertaking line and maintain a strategic distance from the stuffed and insufficient line. Utilize huge practical information from different doctor's facilities to build up a victim therapy time utilization show. Practical victim information are broke down precisely and thoroughly in light of imperative parameters such as victim therapy begin time, end time, persistent age, subtle elements therapy content to every extraordinary undertaking. We recognize, ascertain the distinctive sitting tight time for an alternate patient in light of their conditions and operation performed amid treatment. Therapy time prediction sample is prepared in view of healing facilities authentic information. The holding up time of every therapy errand has anticipated by PTTP and which is aggregate of all patient holding up times in the present line. According to every victim asked for therapy assignments, clinic lining suggestion framework prescribes a proficient and advantageous treatment arrange with the minimum sitting tight time for the patient.

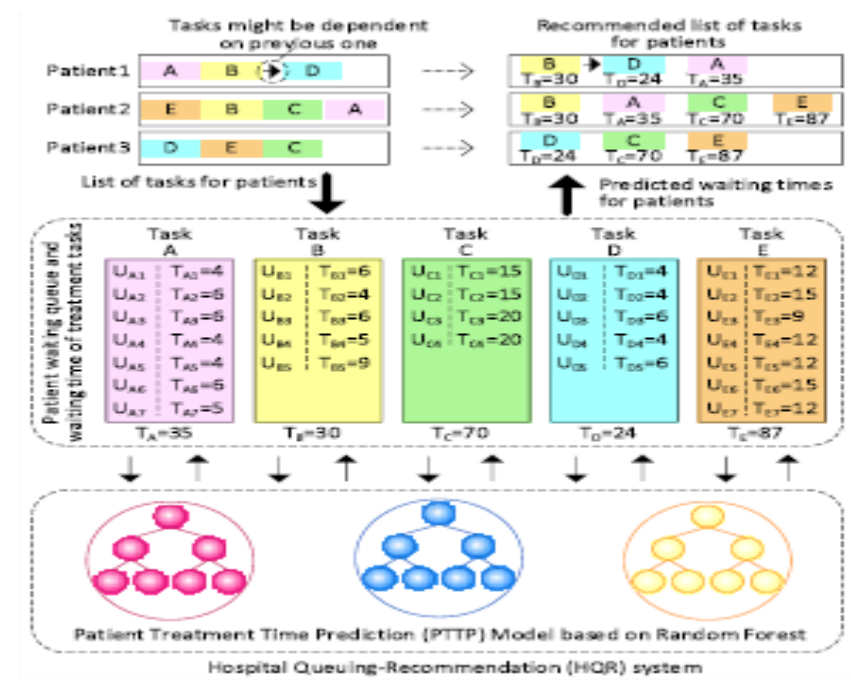

Fig1. System Architecture

\section{EXPERIMENTAL DESIGNS AND RESULTS}

Results exhibit that PTTP and HQR system finish high precision and execution. A treatment proposition with a viable and profitable treatment orchestrate and the scarcest sitting tight time is recommended for each patient 


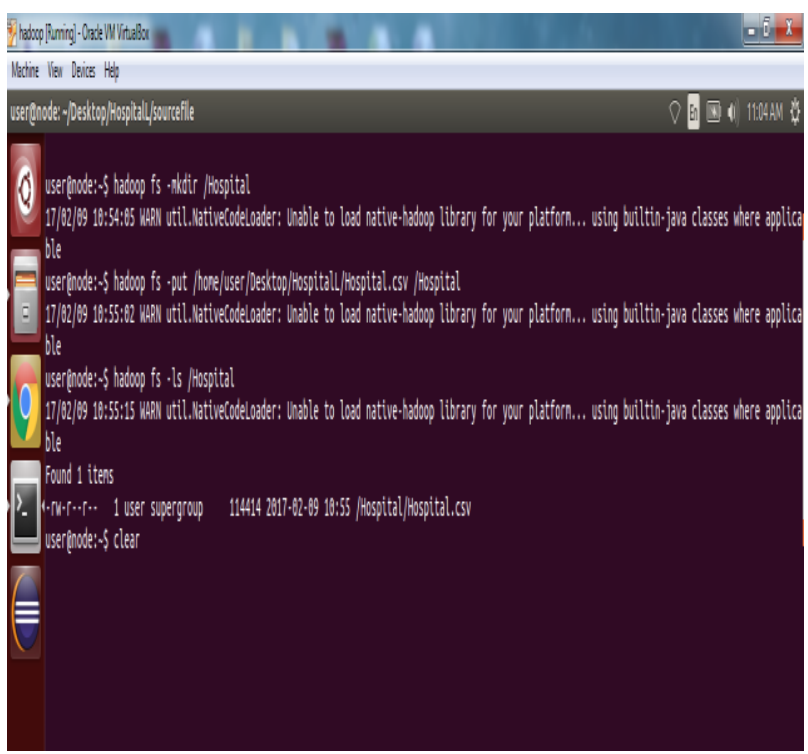

Figure 1: Creating New folder on cloud

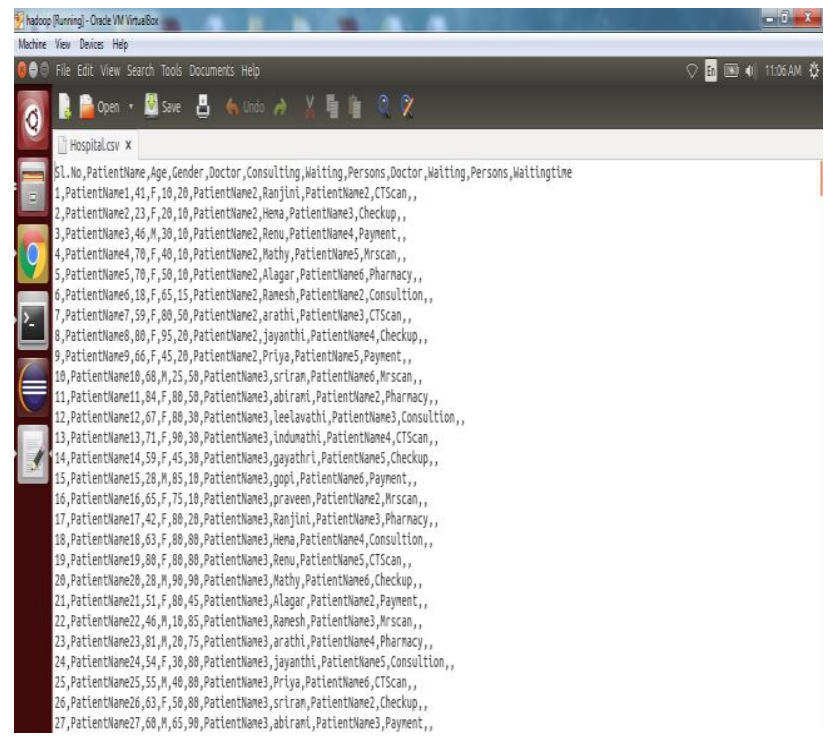

Figure 2: Dataset

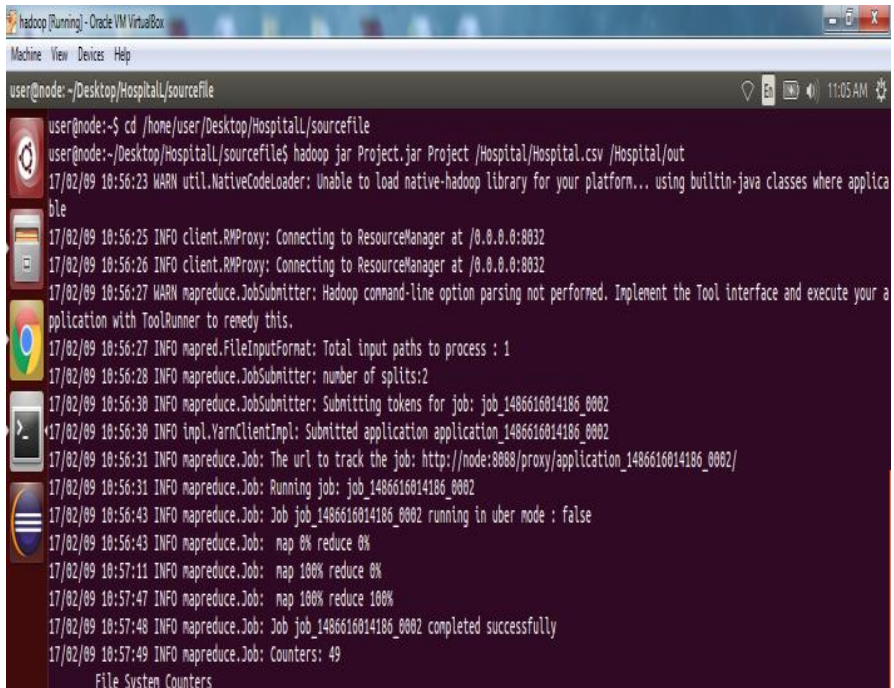

Figure 3: Dataset successful moved to the user location 


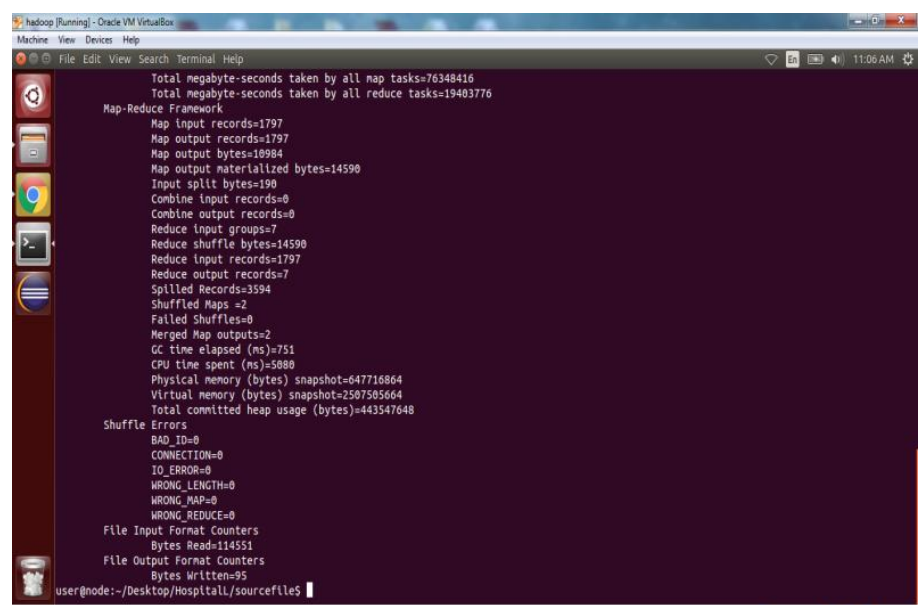

Figure 4: Map and Reduce Task

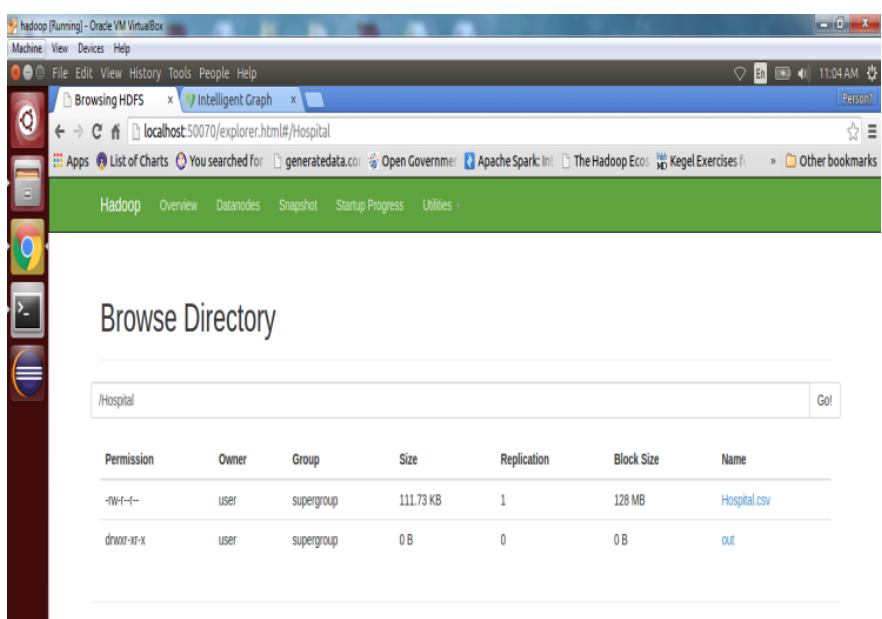

Figure 8.5: Output Folder Successful Created

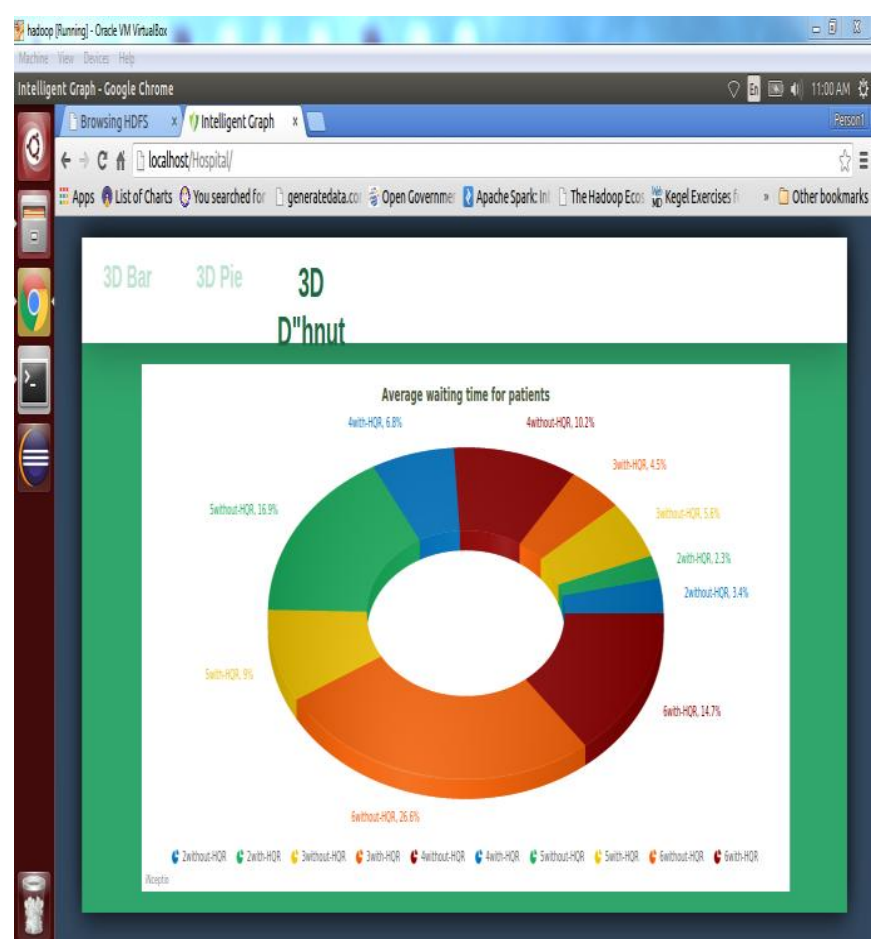

Figure 6: Normal sitting tight time for patients in $3 \mathrm{~d}$ hnut 


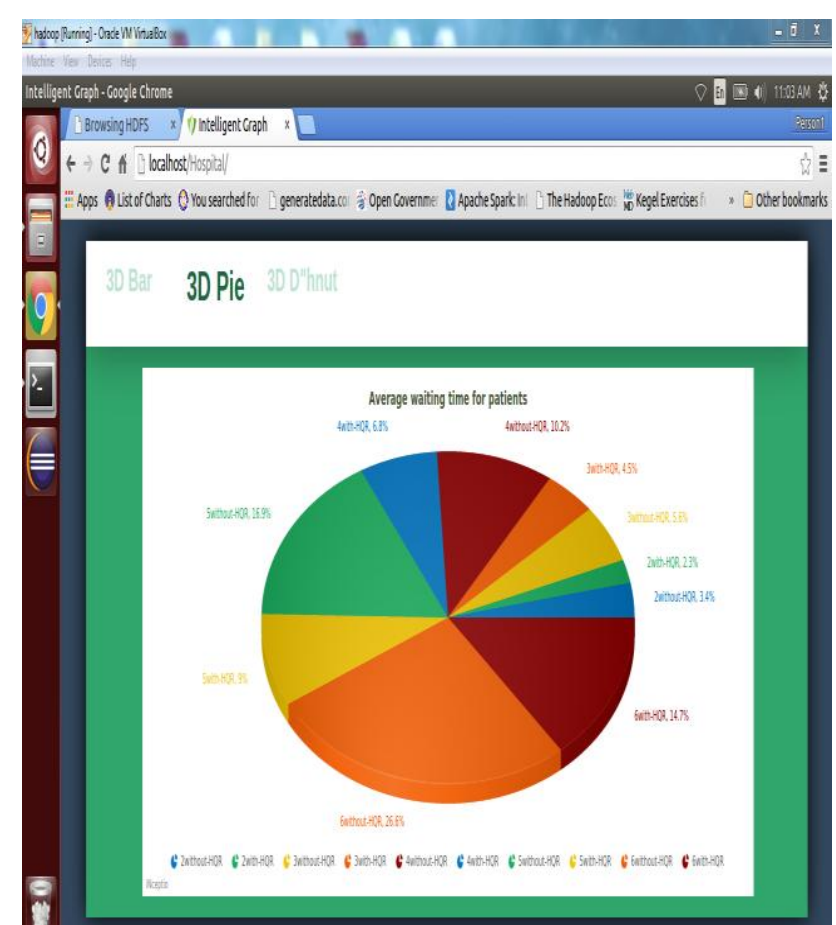

Figure 7: Average sitting tight time for patients in 3D Pie

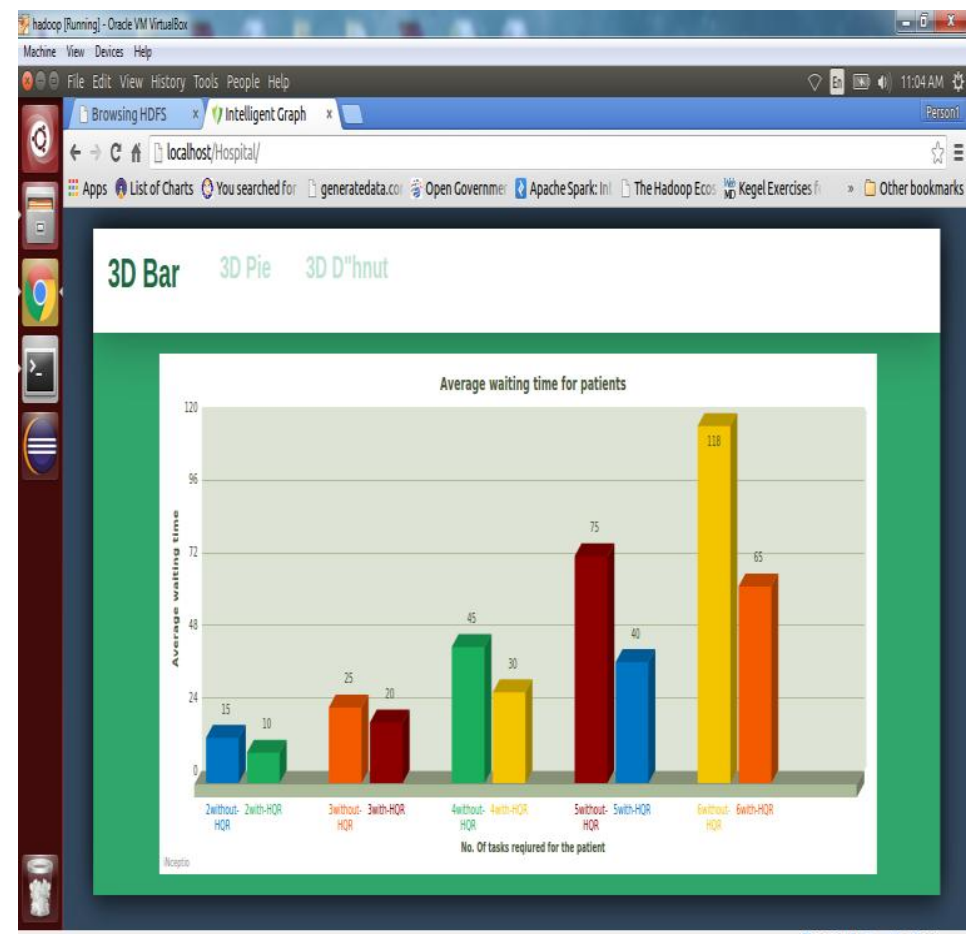

Figure 8: Normal sitting tight time for patients in 3D Bar

\section{CONCLUSION}

PTTP calculation in light of enormous information and the Apache Spark cloud condition is proposed. Unpredictable woodlands streamlining computation are performed to the PTTP show. Line holding up time of each therapy errand is expected in perspective of the arranged PTTP appear. Parallel HQR structure is delivered, efficient and worthwhile therapy plan are prescribed to an individual victim. results demonstrate the PTTP calculation and also HQR framework accomplishes high accuracy, execution.

Healing facilities' information volumes are expanding each day. The workload of setting up recorded information in every game plan mending focus direct proposals is depended upon to be high, yet it requires not be. In 
this way, an incremental PTTP computation in light of spilling data and a more profitable proposition with constrained way care are recommended for future work

\section{REFERENCES}

[1] R. Fidalgo-Merino and M. Nunez, "Self-versatile enlistment of relapse trees," IEEE Trans. Design Anal. Mach. Intell., vol. 33, no. 8, pp. 16591672, Aug. 2011.

[2] S. Tyree, K. Q. Weinberger, K. Agrawal, and J. Paykin, "Parallel supported relapse trees for net hunt positioning," in Proc. twentieth Int. Conf. Overall net (WWW), 2012, pp. 387-396.

[3] N. Salehi-Moghaddami, H. S. Yazdi, and H. Poostchi, "Correlation based generally cacophonic criterionin multi branch call tree," Central Eur. J. Comput. Sci., vol. 1, no. 2, pp. 205-220, Jun. 2011.

[4] G. Chrysos, P. Dagritzikos, I. Papaefstathiou, and A. Dollas, "HC-CART: A parallel framework execution of data mining classi?cation and relapse tree (CART) govern on a multi-FPGA framework," ACM Trans. Archit. Code Optim., vol. 9, no. 4, pp. 47:1-47:25, Jan. 2013.

[5] Y. Ben-Haim and E. Tom-Tov, "A spilling parallel call tree lead," J. Mach. Learn. Res., vol. 11, no. 1, pp. 849-872, Oct. 2010.

[6] L. Breiman, "Random timberlands," Mach. Learn., vol. 45, no. 1, pp. 5-32, Oct. 2001.

[7] G. Yu, N. A. Goussies, J. Yuan, and Z. Liu, "Fast activity identification by means of discriminative arbitrary backwoods balloting and top-K subvolume seek," IEEE Trans. Sight and sound, vol. 13, no. 3, pp. 507-517, Jun. 2011.

[9] K. Singh, S. C. Guntuku, A. Thakur, and C. Hota, "Big data examination structure for distributed botnet identification misuse arbitrary backwoods," Inf. Sci., vol. 278, pp. 488-497, Sep. 2014.

[10] S. Bernard, S. Adam, and L. Heutte, "Dynamic arbitrary woods," Pattern Recognit. Lett., vol. 33, no. 12, pp. 1580-1586, Sep. 2012.

[11] H. B. Li, W. Wang, H. W. Ding, and J. Dong, "Trees coefficient arbitrary woods procedure for arranging high-dimensional shouting information," in Proc. IEEE seventh Int. Conf. e-Business Eng. (ICEBE), Nov. 2010, pp. 160-163. 